\title{
Corona Performance in Wire-cylinder ESP without Particle Loading
}

\author{
Mohamed Th. El-Mohandes ${ }^{1}$, Mazen Abdel-Salam ${ }^{1 . a}$ and Seham Kamal El-deen ${ }^{1}$ \\ ${ }^{1}$ Electrical Engineering Department, Assiut University, Assiut, Egypt
}

\begin{abstract}
This paper is aimed at investigating thoroughly the corona performance in the wirecylinder electrostatic precipitor (ESP) without suspended particles. The onset voltage of negative corona on the discharge wire is calculated based on the criterion of self-sustained discharge. The ionized field in the ESP is mathematically modeled for calculating the spatial distribution of the space-charge density due to the corona ions as well as the components of the electric field including the applied field and the field due to the space charge. This is in addition to the calculation of the current-voltage characteristics of the ESP.
\end{abstract}

\section{Introduction}

The electrostatic precipitator is the most effective device to collect industrialparticulates before escaping into the atmosphere to pollute it. This is why the researchers over the years work on modeling the processes inside the electrostatic precipitors (ESPs)including corona discharge seeking improvement of their performance.

A model was described for computation of charge density and electric field structures without particle loading. The model employed an iterative model based on the finite element method for computing electric potential structure in wire-duct electrostatic precipitators for an assumed charge density distribution by solving Poisson's equation, and the method of characteristics for computing charge density structure for an assumed electric field distribution[1-3].

The charge simulation technique has been adapted [4] tomodel the electrostatic and the corona characteristics in clean air of a duct-type electrostatic precipitator where the corona onset voltage is predicted by Peek's formula [5].

In a wire-duct electrostatic precipitator, the current-voltage characteristics under clean air conditions were predicted [6]. The approach was based on the simultaneous solution for the governing Poisson's and current-continuity equations using a combined boundary element and finite difference method.

An iterative numerical methodology was developed [7] for the calculation of the electric field in wire-duct precipitation systems using finite differencing in orthogonal curvilinear coordinates to solve the potential equation. Charge density distribution was obtained by the method of characteristics or using a prediction-correction scheme. To predict the I-V characteristic of wire-duct ESP which agrees

${ }^{\text {a } C o r r e s p o n d i n g ~ a u t h o r: ~ m a z e n 2000 a s @ y a h o o . c o m . ~}$ 
reasonably with the measured one, an empirically estimated thickness of the ionization zone was considered based on Peek's formula for assessing the corona onset voltage.

In wire-duct ESP, Poisson's equation and the continuity equation of corona current density were solved [8] simultaneously after being transformed into integral form. The calculated spatial distribution of the electric potential and charge density were compared with those obtained by White's formula in wire-cylinder ESP [9].

The current-voltage formula for wire-duct ESP was derived by solving Poisson's equation in one dimension for ion large current density values where the electrostatic field is large compared to the space charge field[10]. The current-voltage formula for wire-duct ESP was also derived for high current density values where the space charge is large[9].

There are several attempts to compute the electric field and I-V characteristics of different ESP geometries including wire-cylinder[8, 10, 11] and wire-ductarrangements[1-4,6-11]. The analysis of the ionized-field in these arrangements is based onboundary element method (BEM)[3], charge simulation method (CSM)[4], finite volume characterization method (FVM)[8], finite element method combined with the method of characteristics (MOC) [1, 2], FDM combined with MOC [7]and BEM combined with FDM [6].

Simplifying assumptions commonly adopted in the ESP modeling which are justified:

1. Kaptsov' assumption which states that the field at the coronating surface remains constant at the corona onset value as determined by Peek's formula[5]irrespective of the value of the applied voltage. The ions emanating from the corona electrode have polarity the same as that of the discharge wire. They tend to diminish the electric field at the electrode surface by a value approximately equal to that due to the excess of applied voltage above the onset value, so the field remains constant at its onset value.

2. The mobility of ions remains constant as their transit time from the discharge wire to the collecting electrode is so small for the mobility to change with the ions' life time.

This motivates the authors to investigate thoroughly the corona performance in the wire-cylinder ESP without loading with suspended particles.

\section{Method of Analysis}

The equations describing the electric field and flow of ions in the ESP are the Poisson's equation for the electric field, the equation for current density and the equation of current continuity[12].

Poisson's equation for the electric field is expressed as:

$$
\nabla . E=\frac{\rho}{\epsilon_{0}}
$$

where $\mathrm{E}$ is the electric field with space charge including corona ions, $\rho$ is the volume charge density of ions and $\epsilon_{\mathrm{o}}$ is permittivity of free space.

The electric field $\mathrm{E}$ is expressed as:

$$
E=E_{f+} E_{s}
$$

where $\mathrm{E}_{\mathrm{f}}$ is the electric field without space charge i.e.; the field due to the applied voltage and $\mathrm{E}_{\mathrm{s}}$ is the field due to space charge of ions.

In a wire-cylinder geometry of the precipitator, the space charge affects only the magnitude but not the direction of the electric field, i.e.;

$$
E=\xi E_{f}
$$

where $\xi$ is a scalar which changes over the space between the discharge wire and the collecting cylinder.

From equations (2) and (3), the field $\mathrm{E}_{\mathrm{s}}$ due to space charge is expressed as:

$$
E_{s}=(\xi-1) E_{f}
$$

The equation of precipitator current density is expressed as:

$$
J=k_{i} \rho_{i} E
$$


where $k_{i}$ is the mobility of corona ions.

Combination of equations (1) and (3) results in the following differential equation, whichdefines the spatial distribution of the scalar $\xi$ over the space between the discharge wire and collecting cylinder:

$$
\frac{d \xi}{d r}=\frac{\rho_{i}}{\epsilon_{0} E_{f}}
$$

The continuity equation of the precipitator current density is expressed as:

$$
\nabla . J=0
$$

Combination of equations (3), (5) and (17) results in the following differential equation:

$$
\frac{d \rho_{i}}{d \xi}=-\left(\frac{\rho_{i}}{\xi}\right)
$$

Combining of equations (6) and (8) results in the following differential equationwhich defines the spatial distribution of the ion charge density $\rho_{i}$ over the space between the discharge wire and collecting cylinder:

$$
\frac{d \rho_{i}}{d r}=-\left(\frac{\rho_{i}}{\xi}\right)\left(\frac{\rho_{i}}{\epsilon_{o} E_{f}}\right)
$$

The differential equations (6) and (9) are integrated along the radial direction to determine the spatial distribution of $\xi$ and $\rho_{\mathrm{i}}$ over the space between the discharge wire and collecting cylinder. The initial conditions for such integrals are (i) $\xi$ at discharge wire is equal to $V_{\mathrm{o}} / \mathrm{V}$, where $\mathrm{V}_{\mathrm{o}}$ is the corona onset voltage and $\mathrm{V}$ is the applied voltage. (ii) The electric field at the surface of the discharge electrode remains constant at the onset value, which is Kaptsov's assumption. The onset value of negative corona in the ESP is calculated based on the criterion of self-recurrence of avalanche growth in the ionization zone surrounding the discharge electrode[13].

\section{Results and Discussions}

\subsection{Performance of ESP without particle loading:}

Figure (1) shows how the electric field E changes along the radial direction from the ionization-zone boundary to the collecting cylinder. It is satisfactory that the calculated spatial distribution of the electric field agrees well with the analytical solutions reported in Appendix 1.

Figure (2) shows the spatial distribution of the electric field $E$ and its components $E_{f}$ and $E_{s}$. The integration value of $\mathrm{E}_{\mathrm{f}}$ along the spacing between the wire and cylinder is $59.98 \mathrm{kV}$ which is almost equal to the applied voltage $\mathrm{V}(=60 \mathrm{kV})$. The integration of $\mathrm{E}_{\mathrm{s}}$ along the spacing between the wire and cylinder is almost equal zero. This is why the integrationof the electric field $\mathrm{E}$ is equal to the applied voltage $\mathrm{V}$.

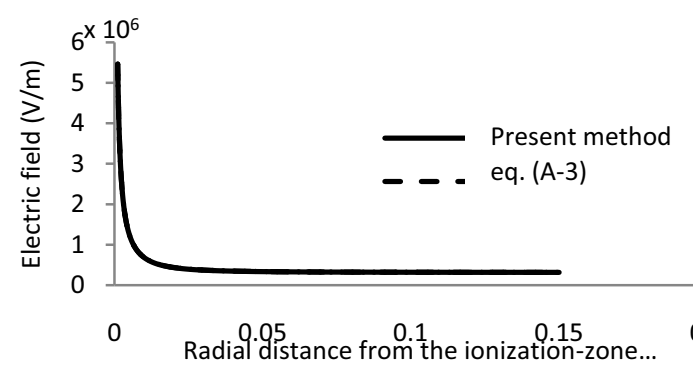

Figure 1: Calculated spatial distribution of the electric field $\mathrm{E}$ between the discharge wire and collecting cylinder compared with that obtained by the analytical equations $(\mathrm{A}-3)$ and $(\mathrm{A}-5) \cdot \mathrm{r}_{\mathrm{o}}=1 \mathrm{~mm}$, $\mathrm{R}=15 \mathrm{~cm}, \mathrm{~V}_{\mathrm{o}}=30.659 \mathrm{kV}$ and $\mathrm{V}=60 \mathrm{kV}$.



Figure 2: Calculated spatial distribution of the electric field $\mathrm{E}$ and its components $\mathrm{E}_{\mathrm{f}}$ and $\mathrm{E}_{\mathrm{s}}$ between the discharge wire and collecting cylinder. $\mathrm{r}_{\mathrm{o}}=1 \mathrm{~mm}, \mathrm{R}=15$ $\mathrm{cm}, \mathrm{V}=60 \mathrm{kV}$ and $\mathrm{Vo}=30.659 \mathrm{kV}$ 
As the electron ionization coefficient increases with the electric field, the ion charge density $\rho_{\mathrm{i}}$ also increases with the increase of the electric field. Therefore, $\rho_{\mathrm{i}}$ assumes high values near the discharge wire and decreases gradually toward the cylinder as shown in Fig. (3). It is satisfactory that the calculated spatial distribution of $\rho_{\mathrm{i}}$ between the wire and cylinder agrees well with the analytical solution eq. (A-4) reported in Appendix 1. The deviation between the present calculated values and those predicted by the analytical solution does not exceed $0.01 \%$.

The calculated current-voltage characteristic of the ESP agrees satisfactory with that predicted by the analytical solution as shown in Fig. (4). The deviation between the calculated and predicted values of corona current at the same applied voltage does not exceed $2.2 \%$.
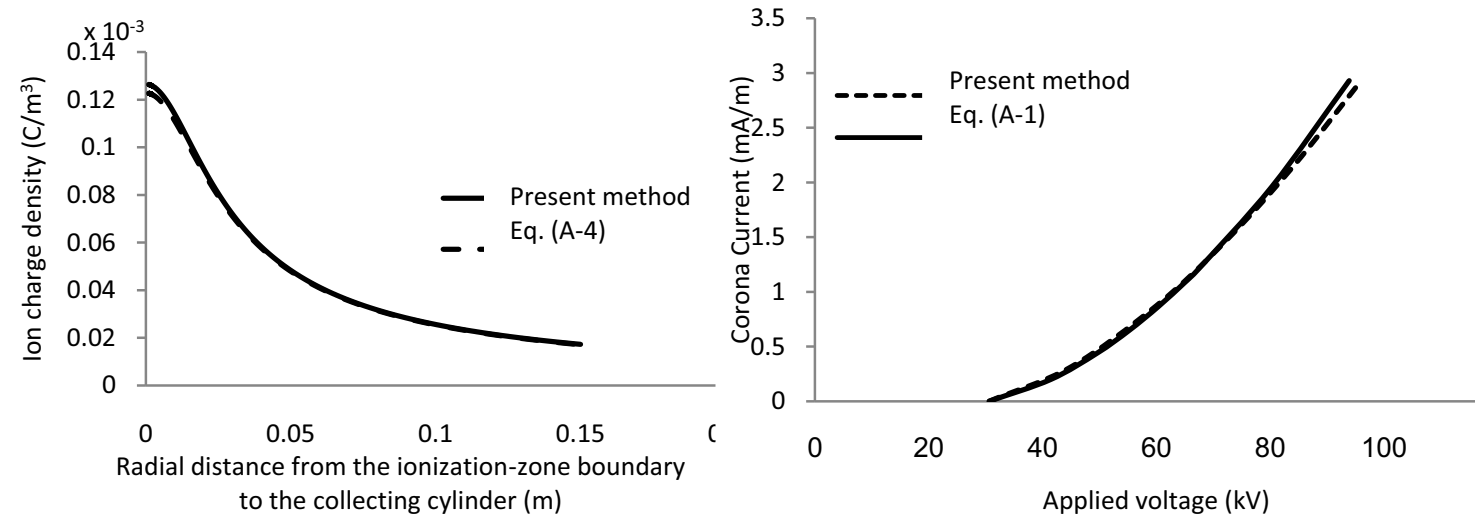

Figure 3: Calculated spatial distribution of the ion charge density between the discharge wire and collecting cylinder compared with that obtained by the analytical equation (A-4). $\mathrm{r}_{\mathrm{o}}=1 \mathrm{~mm}, \mathrm{R}=$ $15 \mathrm{~cm}, \mathrm{~V}_{\mathrm{o}}=30.659 \mathrm{kV}$ and $\mathrm{V}=60 \mathrm{kV}$.

Figure 4: Calculated corona current-voltage characteristics of the ESP compared with that obtained by the analytical equation (A-1) for wide range of the corona current. $\mathrm{r}_{\mathrm{o}}=1 \mathrm{~mm}, \mathrm{R}=15 \mathrm{~cm}$ and $\mathrm{V}_{\mathrm{o}}=30.659 \mathrm{kV}$

Figure (5) shows the current-voltage (I-V) characteristics of the ESP at varying radius $r_{o}$ of the discharge wire for the same radius $\mathrm{R}$ of the collecting cylinder. The onset voltage increases with the increase of the discharge wire $r_{o}$ as given in Table (1) for the same radius $R$ of the cylinder.

Figure (6) shows the I-V characteristics of the ESP at varying radius $\mathrm{R}$ of the collecting cylinder for the same radius $r_{0}$ of the discharge wire. The onset voltage decreases with the increase of $R$ for the same radius $r_{o}$ as given in Table (2).
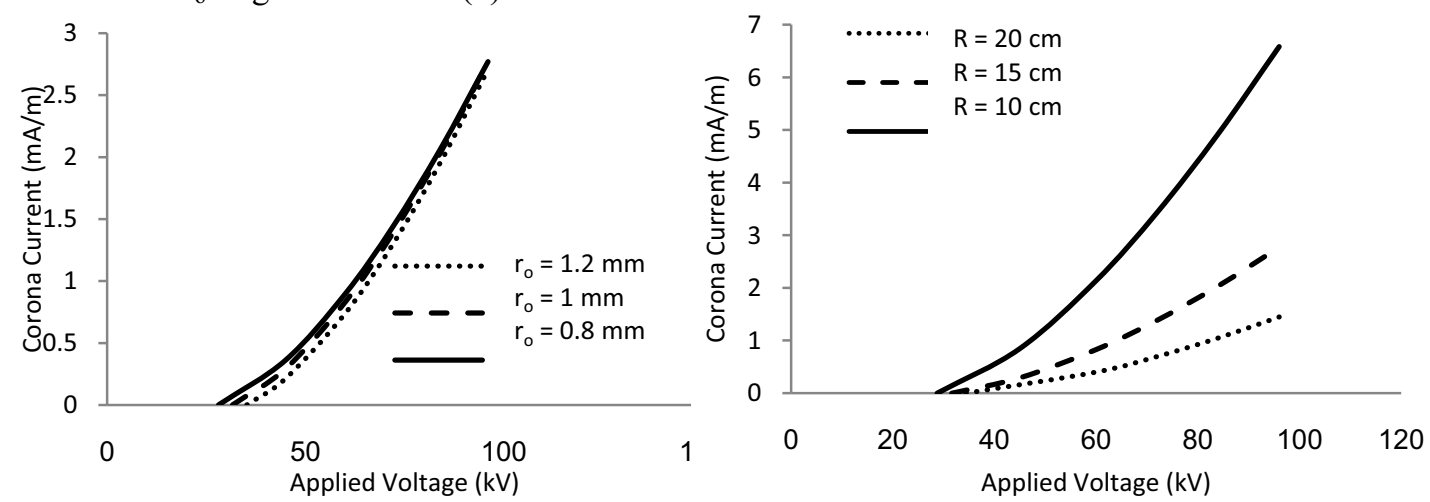

Figure 5: Calculated corona I-V characteristics of the ESP for different values of the discharge wire radius $r_{o}$ at the same radius $R(=15 \mathrm{~cm})$ of

Figure 6: Calculated corona I-V characteristics of the ESP for different values of the collecting-cylinder radius $\mathrm{R}$ at the same radius of the discharge wire $r_{o}(=1 \mathrm{~mm})$ the collecting cylinder. 
Table 1: Calculated corona onset voltage $V_{o}$ as influenced by the radius of the discharge wire at the same radius $\mathrm{R}(=15 \mathrm{~cm})$ of the collecting cylinder.

\begin{tabular}{|c|c|c|c|}
\hline $\mathrm{r}_{\mathrm{o}}(\mathrm{mm})$ & 0.8 & 1 & 1.2 \\
\hline $\mathrm{V}_{\mathrm{o}}(\mathrm{kV})$ & 28.05942 & 31.57891 & 35.21861 \\
\hline
\end{tabular}

Table 2: Calculated corona onset voltage $V_{o}$ as influenced by the radius of the collecting cylinder $\mathrm{R}$ at the same radius of the discharge wire $r_{o}(=1 \mathrm{~mm})$.

\begin{tabular}{|c|c|c|c|}
\hline $\mathrm{R}(\mathrm{cm})$ & 10 & 15 & 20 \\
\hline $\mathrm{V}_{\mathrm{o}}(\mathrm{kV})$ & 28.74174 & 31.57891 & 34.36458 \\
\hline
\end{tabular}

\section{Conclusions}

1. The onset voltage of negative corona on the dischargewire of the ESP is calculated based on the criterion ofself-sustained discharge instead of using the empirical equation of Peek.

2. The ionized field in the ESP is mathematically modeled for calculating the spatial distribution ofspace charge density due to corona ions as well as the components of electric field including the applied field and the field due to space charge.

3. The calculated spatial distribution of the electric field between the discharge wire and cylinder of the ESP without particle loading agreed very well with that obtained analytically.

4. The integration of the electric field with and without space charge is equal to the applied voltage with a deviation not exceeding $0.01 \%$. This reflects itself on the accuracy of the present method of calculation.

5. The calculated spatial distribution of the ion charge density over the space between the discharge wire and cylinder agreed reasonably with that obtained analytically.

6. The calculated current-voltage characteristics of the ESP without particle loading agreed reasonably with that obtained analytically.

7. The calculated onset voltage of corona on the discharge wire of the ESP without particle loading increases with the increase of either the wire radius or the cylinder radius.

\section{Acknowledgment}

This paper is extracted from a research project sponsored through joint innovative projects fund STDF(Egypt) - IRD (France), project STDF ID-5457. The authors acknowledge such sponsorship.

\section{References}

1. J. L. Davis and J. F. Hoburg, "Wire-duct precipitator field and charge computation using finite element and characteristics methods," J. Electrostat., 14, 2(1983).

2. A. J. Butler, Z. J. Cendes, and J. F. Hoburg, "Interfacing the finite-element method with the method of characteristics in self-consistent electrostatic field models," IEEE Trans. Ind. Appl., IA-25, 3(1989).

3. K. Adamiak, "Simulation of Corona in Wire-Duct Electrostatic Precipitator by Means of the Boundary Element Method," IEEE Trans. Ind. Appl. vol. IA-30, no. 2, pp. 381-386, 1994.

4. A. A. ElMoursi and G. S. P. Castle, "Modeling Corona Characteristics in a Wire-Duct Precipitator Using the Charge Simulation," IEEE Trans. on Ind. Appl., IA-23, 1(1987).

5. F.W. Peek, "Dielectric Phenomena in High-Voltage Engineering," McGraw-Hill, New Work, USA, (1929).

6. B. S. Rajanikanth and N. Thirumaran, "Prediction of pre-breakdown V-I characteristics of an electrostatic precipitator using a combined boundary element-finite difference approach," Fuel Process. Technol., 76, 3(2002).

7. J. Anagnostopoulos and G. Bergeles, "Corona discharge simulation in wire-duct electrostatic precipitator," J. Electrostat., 54, 2(2002).

8. N. Neimarlija, I. Demirdžić, and S. Muzaferija, "Finite volume method for calculation of electrostatic fields in electrostatic precipitators," J. Electrostat., 67, 1(2009). 
9. H. J. White, Industrial electrostatic precipitation. Reading, MA: Addison-Wesley, (1963).

10. P. Cooperman, "A theory for space charge limited currents with application to electrical precipitation," AIEE Trans., 79, 47-50(1960).

11. A. D. Moore, "Electrostatics and its Applications", J. Wiley \& Sons, New York, USA, (1973).

12. M. Abdel-Salam and Z. Al-Hamouz, "A New Finite Element Solution of Ionized Field in Coaxial Cylindrical Geometry", J. Phys. D: Applied Physics, 25, 1551-1555(1992).

13. M. Abdel-Salam and D. Wiitanen, "Calculation of corona onset voltage for duct-type precipitators,” IEEE Trans. Ind. Appl., IA-29, 2(1993).

14. M. P. Sarma and W. Janischewskyj And, "Analysis of Corona Losses on DC Transmission Lines: I - Unipolar Lines,” IEEE Trans. Power Appar. Syst., PAS-88, 718-731(1969).

\section{Appendices}

\subsection{Appendix 1: Analytical expressions defining wire-cylinder ESP performance without particle loading:}

\subsubsection{Discharge current-voltage characteristics:}

The characteristics were expressed in the literature by equation (A-1) [14].

where:

$$
\frac{\mathrm{V}-\mathrm{V}_{\mathrm{o}}}{\mathrm{V}_{\mathrm{o}}} \ln \frac{\mathrm{R}}{\mathrm{r}_{\mathrm{o}}}=\sqrt{(1+\mathrm{Y})}-1+\ln \frac{2}{1+\sqrt{1+\mathrm{Y}}}
$$

$\mathrm{I}$ is the current per unit length.

$$
\mathrm{Y}=\frac{\mathrm{I}}{2 \pi \epsilon_{\mathrm{o}} \mathrm{K}}\left(\frac{\mathrm{R}}{\mathrm{E}_{\mathrm{o}} \mathrm{r}_{\mathrm{o}}}\right)(\mathrm{A}-1 \mathrm{~b})
$$

\subsubsection{Spatial distribution of electric potential $\phi$, electric field $E$ and ion charge density $\rho_{i}$ :}

The expressions (A-2) and (A-4) were reported before for the potential $\phi(\mathrm{r})$ and ion charge density $\rho \mathrm{i}$ along the radial distance $\mathrm{r}$ from the discharge wire axis [9]:

$$
\phi(r)=\sqrt{\left(\frac{I^{2}}{2 \pi \epsilon_{0}}+A^{2}\right)}+\sqrt{\left(\frac{I R^{2}}{2 \pi \epsilon_{0}}+A^{2}\right)}+A \ln \left(\frac{A+\sqrt{\frac{I R^{2}}{2 \pi \epsilon_{0}}+A^{2}}}{A+\sqrt{\frac{I r^{2}}{2 \pi \epsilon_{0}}+A^{2}}}\right)+A \ln \frac{r}{R}
$$

where:

$$
A=r_{o} \sqrt{E_{o}^{2}-\frac{I}{2 \pi \epsilon_{0} K}}
$$

The electric field $E(r)$ is obtained as equal to $\frac{d(\phi(r))}{d r}$ :

$$
\begin{aligned}
& \mathrm{E}(\mathrm{r})=\frac{\mathrm{Ir} / \pi \epsilon_{\mathrm{o}} \mathrm{k}_{\mathrm{i}}}{2 \sqrt{\frac{\mathrm{Ir} \epsilon^{2}}{2 \pi \epsilon_{\mathrm{o}}}+\mathrm{A}^{2}}}+\mathrm{A}\left[\frac{-\mathrm{Ir} / \pi \epsilon_{\mathrm{o}} \mathrm{k}}{2\left(\mathrm{~A}^{2}+\sqrt{\frac{\mathrm{Ir}{ }^{2}}{2 \pi \epsilon_{\mathrm{o}}}+\mathrm{A}^{2}}\right)\left(\sqrt{\frac{\mathrm{Ir}}{2 \pi \epsilon_{\mathrm{o}}}+\mathrm{A}^{2}}\right)}\right]+\frac{\mathrm{A}}{\mathrm{r}} \\
& \rho_{i}(r)=\frac{I}{2 \pi r k_{i}} \frac{1}{\sqrt{\frac{I}{2 \pi \epsilon_{0} K}\left(1-\frac{r_{0}^{2}}{r^{2}}\right)+\frac{r_{0}^{2}}{r^{2}} E_{i}^{2}}}
\end{aligned}
$$

\subsubsection{Spatial distribution of electric field $E$}

The spatial distribution of electric field E (r) was expressed before [11]:

$$
E(r)=\left(\frac{I}{2 \pi \epsilon_{0} k_{i}}+\left(\frac{r_{0}}{r}\right)^{2}\left(E_{o}^{2}-\frac{I}{2 \pi \epsilon_{0} k_{i}}\right)\right)^{1 / 2}
$$

